\title{
MAGNETO-OPTICAL AND MAGNETIC PROPERTIES OF EXCHANGE-COUPLED TbNdFeCo FILMS
}

\author{
Y. FUJII, K. HASHIMA* and K. TSUTSUMI* \\ Industrial Electronics \& Systems Development Lab., \\ *Materials \& Electronic Devices Lab., Mitsubishi Electric Corp., 8-1-1 Tsukaguchi-honmachi, Amagasaki, Hyogo 661, Japan
}

\begin{abstract}
Magneto-optical and magnetic properties of single and exchange-coupled double-layered TbNdFeCo films were examined. Very thin TbNdFeCo films with small Tb content were found to be suitable as the 1st layer of double-layered TbNdFeCo films. Large coercivity and large Kerr rotation were concurrently realized in exchange-coupled double-layered TbNdFeCo films. It was also shown that the exchange-coupled doublelayered TbNdFeCo film has a good recording characteristics at the wavelength of 830nm and the potentiality as a recording medium for shorter wavelength is suggested. KEYWORDS : TbNdFeCo, MAGNETO OPTICAL DISK, EXCHANGE COUPLING, DOUBLE LAYER
\end{abstract}

\section{INTRODUCTION}

TbNdFeCo film is one of the candidates for future magneto-optical $(M-0)$ recording media for shorter wavelength light source. In applying TbNdFeco film to practical use, the main problem is its smaller coercivity than that of TbFeCo film [1]. Large Hc and large Kerr rotation angle $\theta k$ seem to be irreconcilable demands. One solution is to divide the magnetic layer into two layers, one of which has larger $\theta \mathrm{k}$ and the other has larger Hc. We presented exchange-coupled double-layer TbFeco films as a high performance recording medium [2][3]. In this study, we examined the magneto-optical and the magnetic properties of TbNdFeCo films and exchange-coupled double-layered very thin TbNdFeco films in order to obtain a feasible recording medium.

\section{EXPERIMENTAL PROCEDURE}

TbNaFeco films were sputter-deposited from Feco target with Nd pellets and $T b$ target onto rotating glass substrates by a dc planer magnetron sputtering method. Double-layered TbNdFeCo films with SiN $_{x}$ dielectric layers and Al reflective layer shown in fig.1 were sputter-deposited successively on a glass or a pregrooved polycarbonate ( $P C$ ) substrate. Transitionmetal(TM) dominant and rare-earth(RE) dominant compositions were chosen for the 1st and the and TbNdFeco layer, respectively.

\begin{tabular}{|c|l}
\hline Substrate & \multirow{2}{*}{ PC or Glass } \\
Dielectric Layer & SiNx \\
Magnetic Layer 1 & TbNdFeCo \\
Magnetic Layer 2 & TbNdFeCo \\
Dielectric Layer & SiNx \\
\cline { 1 - 1 } Reflective Layer & Al \\
\cline { 1 - 1 } Protective Coat. & Resin \\
\hline
\end{tabular}

Fig. 1 Sample configuration

Reflectivity $R$ and Kerr rotation $\theta \dot{k}$ were measured in the wayelength range of 400-830nm. Kerr hysteresis loop were obtained in maximum field range of $15 \mathrm{k} 0 \mathrm{e}$. The saturation magnetizations Ms were measured by the vibrating sample magnetometer.

\section{CHARACTERISTICS OF TbNdFeCo FILMS}

Fig.2 shows the wavelength dependence of $\theta \mathrm{k}$ measured from the film sides of $\mathrm{TbNd}-$ FeCo films whose thicknesses are 1200A. Ms and $\mathrm{Hc}$ values of these samples are shown in table 1. $\theta \mathrm{k}$ increases as the wavelength is shortened and exceeds that of TbFeCo film which has the almost compensation composition. Tb addition decreases $\theta k$ in the whole wavelength range, which agrees to Reim's results[1]. It is confirmed that to make the most use of the advantage of large $\theta k$ 
at shorter wavelength in TbNdFeCo film, Tb addition should be kept at a minimum.

On the other hand, TbNdFeco films with small Tb content show Kerr hysteresis loops with poor squareness because of their large Ms values. Cape et al. showed that thinner garnet film has smaller saturation field Hs than that of thicker one and gave the theoretical interpretation [4]. We examined film thickness dependence of hysteresis loops of TbNdFeCo films. Fig.3 shows the results. Saturation field Hs decreases with decreasing the film thickness, and the almost perfect square loop is obtained at the thickness less than about $200 \AA$. Solid curves in the figure show calculated Hs for several values of Bloch wall energy assuming the stripe domain configuration. It agrees very well to measured Hs when the Bloch wall energy is $0.5 \mathrm{erg} / \mathrm{cm}^{2}$.

As a result from above experiments, very thin TbNdFeco film of small $\mathrm{Tb}$ content is preferable as the 1st layer of doublelayered $T$ bNdFeco film for a recording medium.

Table 1 Ms and Hc of samples in fig.2

\begin{tabular}{|lcrc|}
\hline Tb content & Ms & Hc \\
\hline$X=0$ atx & 580 emu/cc & 310 Oe \\
$X=8$ at $x$ & 260 emu/cc & 860 Oe \\
$X=16$ atx & $170 \mathrm{emu} / \mathrm{cc}$ & 1120 Oe \\
\hline
\end{tabular}

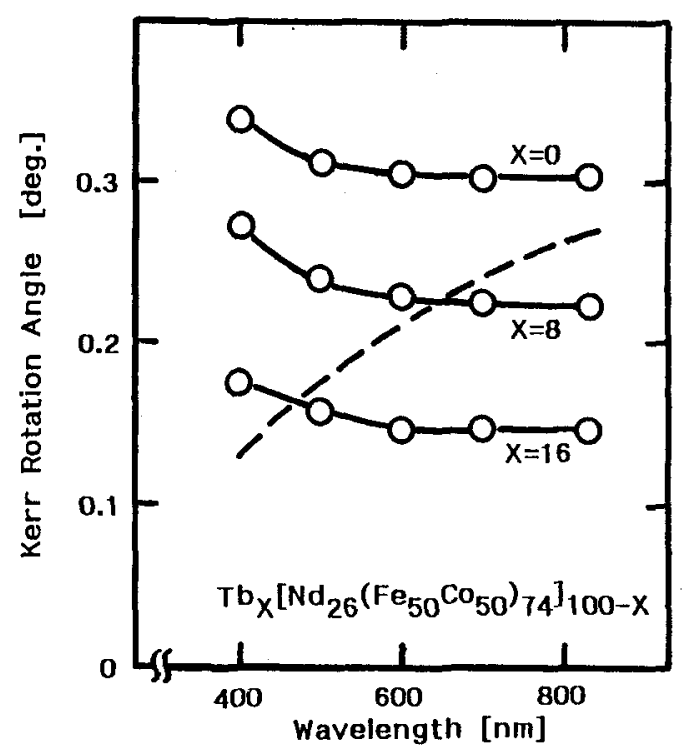

Fig.2 Wavelength dependence of Kerr rotation angle of TbNdFeCo films. Dashed curve : $\mathrm{Tb}_{21}\left(\mathrm{Fe}_{90} \mathrm{Co}_{10}\right)_{79}$

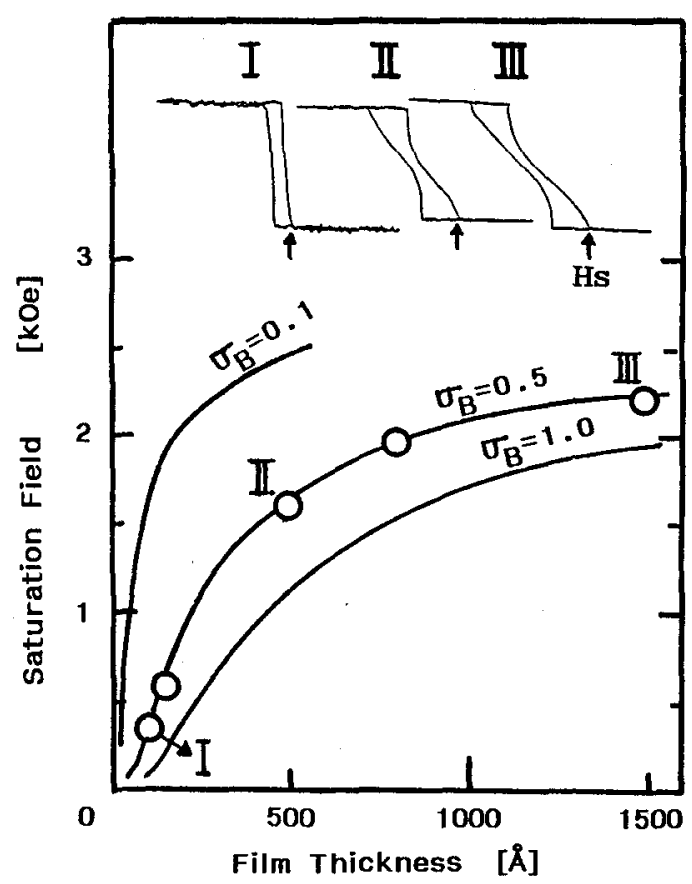

Pig.3 Thickness dependence of Kerr loop and saturation field Hs of TbNdFeCo film. Solid curves : calculated Hs for several Bloch wall energy $\sigma_{B}\left[\mathrm{erg} / \mathrm{cm}^{2}\right]$.

\section{DOUBLE-LAYERED TbNdFeCo FILMS}

\section{Magnetic characteristics}

The 1st and the 2nd TbNdFeCo layer are coupled magnetically by the exchangecoupling and it affects the hysteresis loop of the film. Two kinds of Kerr loops of exchange-coupled double-layered TbNdFeCo films were observed,which is shown as sample $A$ and $B$ in fig.4. These samples are the same except the thickness of the 2 nd TbNdFeCo

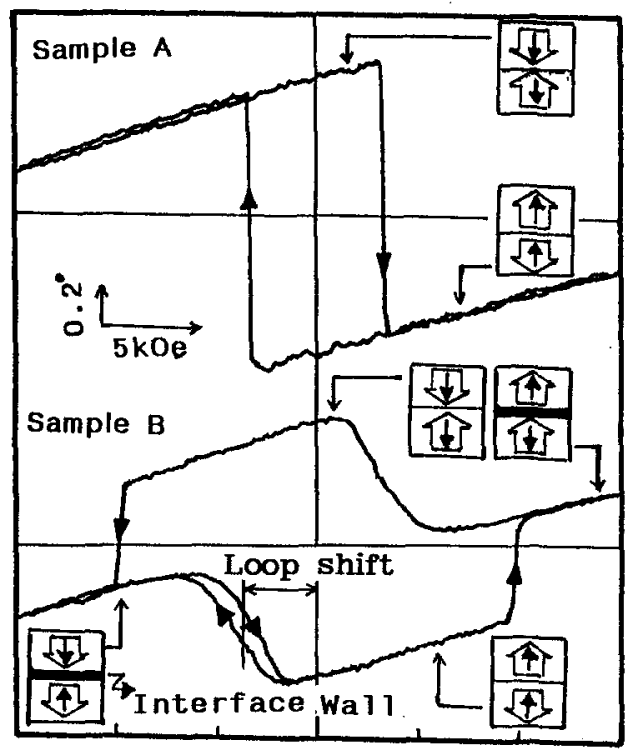

Fig.4 Kerr loops of exchange-coupled double-layered TbNdFeCo films. 
Table $2 \mathrm{Ms}$, He and $t$ of sample $A$ and $B$

\begin{tabular}{|c|c|c|c|}
\hline Sample: & $\begin{array}{l}\text { Ms1 Ms2 } \\
{[\mathrm{emu} / \mathrm{cc}]}\end{array}$ & $\begin{array}{c}\mathrm{Hc1} \mathrm{Hc2} \\
] \quad[\mathrm{Oe}]\end{array}$ & $\mathrm{t1}_{[\AA]}^{\mathrm{t} 2}$ \\
\hline $\mathbf{A}$ & 490290 & 2401800 & 100100 \\
\hline B : & $490 \quad 290$ & 2401800 & 100200 \\
\hline
\end{tabular}

layer. Magnetization direction ( $5 \cdot 5$ ) and TM sublattice moment direction $(\uparrow \cdot \downarrow)$ at each state of the loops are also illustrated in fig.4.

Sample A shows perfectly square hysteresis loop. The 1st and the 2nd layer reverses at the same field and no interface wall appears within measured field range. On the other hand, the 1st layer of sample $B$ reverses alone and its hysteresis loop is shifted and slanted. Switching field of sample $A$ and the loop shift of the 1st layer of sample $B$ can be expressed respectively as

$$
\frac{M s 1 \cdot t 1 \cdot H c 1+M s 2 \cdot t 2 \cdot H c 2}{M s 1 \cdot t 1-M s 2 \cdot t 2}
$$

and

$$
\frac{\sigma w}{2 \cdot M s 1 \cdot t 1}
$$

where Ms, $t$, Hc and $\sigma_{w}$ are saturation magnetization, thickness, coercivity and interface wall energy, respectively, and suffix 1 and 2 represent the 1st and the 2nd TbNdFeCo layer. Each value is summarized in table 2. From these values and equation (1), the switching field of sample $A$ is calculated to be $3.2 \mathrm{kOe}$ which agrees well to the measured value of $3.5 \mathrm{kOe}$. From the loop shift value of the 1st layer of sample $B$ of $3.8 \mathrm{kOe}$, and equation (2), the interface wall energy $\sigma w$ is calculated to be $3.7 \mathrm{erg} / \mathrm{cm}^{2}$ which is slightly smaller than that of the exchangecoupled double-layered TbFeCo film of $5 \mathrm{erg} / \mathrm{cm}^{2}[2]$.

\section{M-O AND RECORDING CHARACTERISTICS}

Fig.5 shows wavelength dependence of the ratio of $R \cdot \theta k$ of exchange-coupled TbNdFeco film (sample C) to that of TbFeCo film (sample D) at each wavelength. $R$ and $\theta k$ were measured from the glass substrate side. Sample compositions are shown in table 3 . Refractive index of $\operatorname{siN}_{x}$ film was measured in advance by ellipsometry and no absorption was observed through the wavelength range of 400-830nm.
Large enhancement in $R \cdot \theta k$ were observed at the wavelength around $400 \mathrm{~nm}$. This is supposed to be attributed to large $\theta k$ of the 1st TbNdFeco layer because the reflectivity of two samples were almost same at each wavelength. From this result, the exchangecoupled double-layered TbNdFeCo medium is expected to have larger read-out signal than TbFeCo medium around $400 \mathrm{~nm}$ wavelength.

In order to estimate the potentiality of the exchange-coupled TbNdFeCo film as a recording medium,we measured recording characteristics at the wavelength of $830 \mathrm{~nm}$ of two disks deposited on PC substrate simultaneously with sample $C$ and $D$. Measurement conditions are summarized in table 4 .

Table 3 Compositions of sample C and D

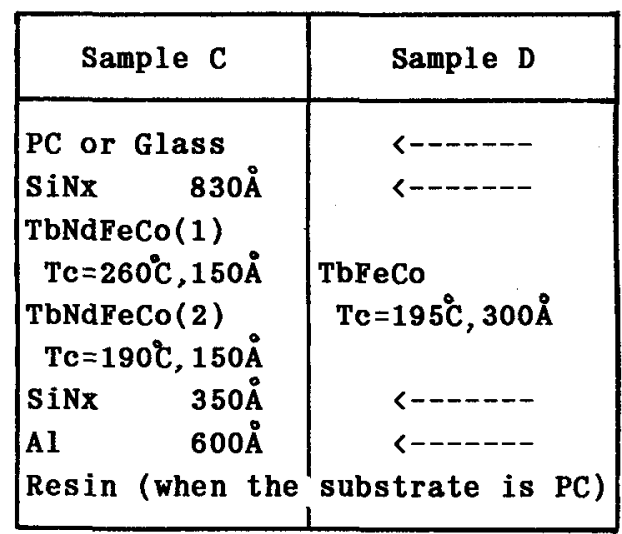

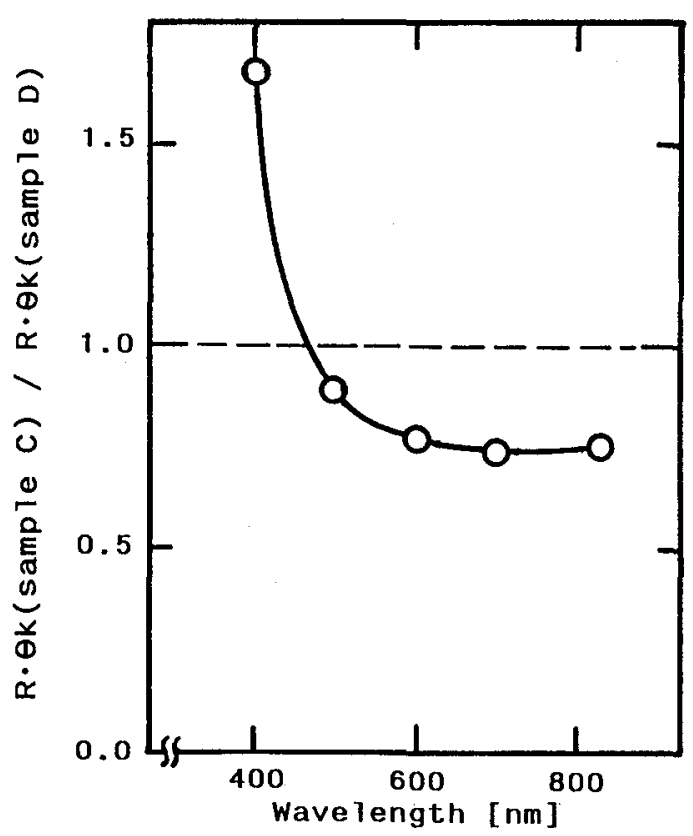

Fig.5 Wavelength dependence of ratio of $R \cdot \theta k$ of double-layered TbNdFeCo film (sample C) to that of TbFeCo film (sample D). 
Fig.6 shows recording laser power dependence of the carrier and noise level of two disks. Both carrier and noise level of sample $C$ are smaller than that of sample $D$. Carrier to noise ratio are almost same for two samples. The difference in carrier level is ascribed to that $R \cdot \theta k$ of sample $C$ is smaller than that of sample $D$ at the wavelength of $830 \mathrm{~nm}$ as shown in fig.5. Carrier level of sample $C$ is expected to surpass that of sample $D$ at shorter wavelength range, e.g. $400 \mathrm{~nm}$, when $R \cdot \theta k$ value exceeds 1.0 in fig.5.

Recording sensitivities of two samples are the same although sample $C$ includes the layer with high Curie temperature. We measured Kerr loops at raised temperature to examine this. Fig.7 shows temperature dependence of $\mathrm{HC}$ of two samples. At $200^{\circ} \mathrm{C}$, HC of sample $C$ is below 2000e and Kerr hysteresis loop keeps squareness as shown in fig.8. Therefore, thermo-magnetic writing on sample $C$ is supposed to take place at around $20{ }^{\circ} \mathrm{C}$ which is near the Curie temperature of sample D.

\section{CONCLUSION}

Magneto-optical and magnetic properties of single and exchange-coupled doublelayered TbNdFeco films were studied. TbNdFeco film, which has large $\theta k$ at shorter wavelength and also has small Hc and large Ms, were utilized as the 1st layer of exchange-coupled film, and it was confirmed that the Hc can be substantially increased by the exchange-coupling. And the exchangecoupled TbNdFeCo film has a fairly good recording characteristics equivalent to that of TbFeco films at the wavelength of $830 \mathrm{~nm}$, and has a potentiality for shorter wavelength applications.

\section{REFERENCES}

[1]W.Rein et al., J.Appl.Phys., 61, No.8,Apr. (1987), 3349.

[2]Y.Fujii et al., J.Magn. Soc.Jpn.,11, Suppl. No. $S 1,(1987), 329$.

[3]H.Sugahara et al., IEEE Trans.Magn., MAG-24 No. $6,(1988), 2455$.

[4]J.A.Cape et al., J. Appl. Phys., 42, No.13 Dec. (1971), 5732 .
Table 4 Measurement conditions
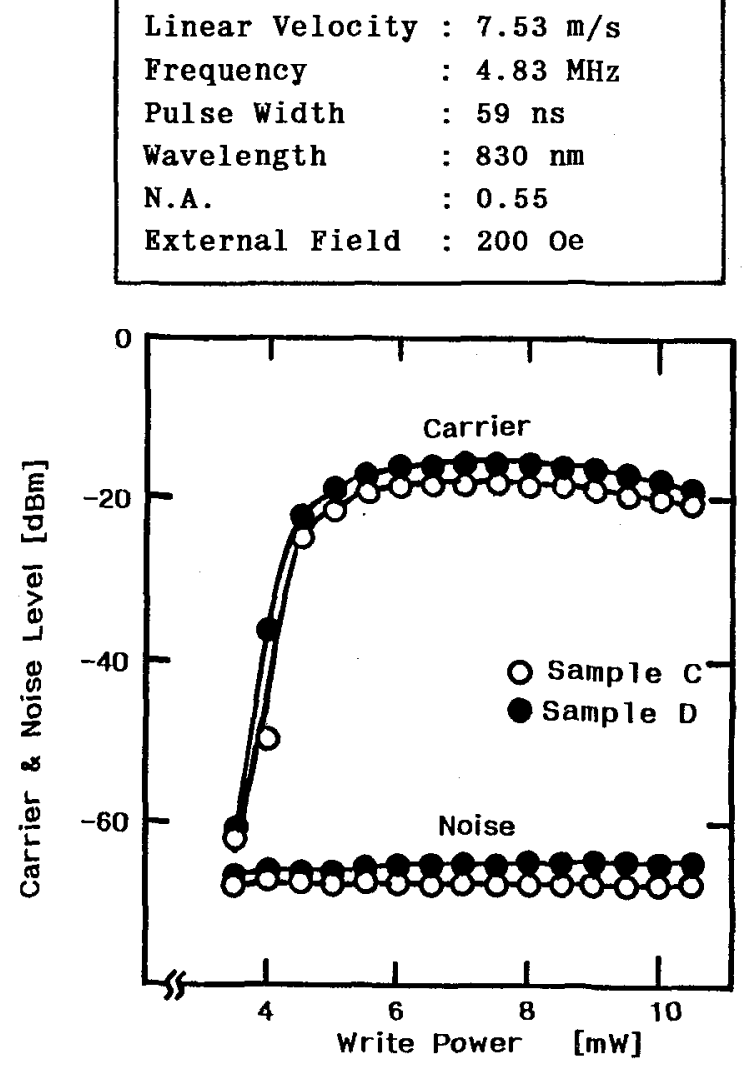

Fig.6 Write power dependence of carrier and noise of Sample C: double-layered TbNdFeCo Sample D:single-layered TbFeCo

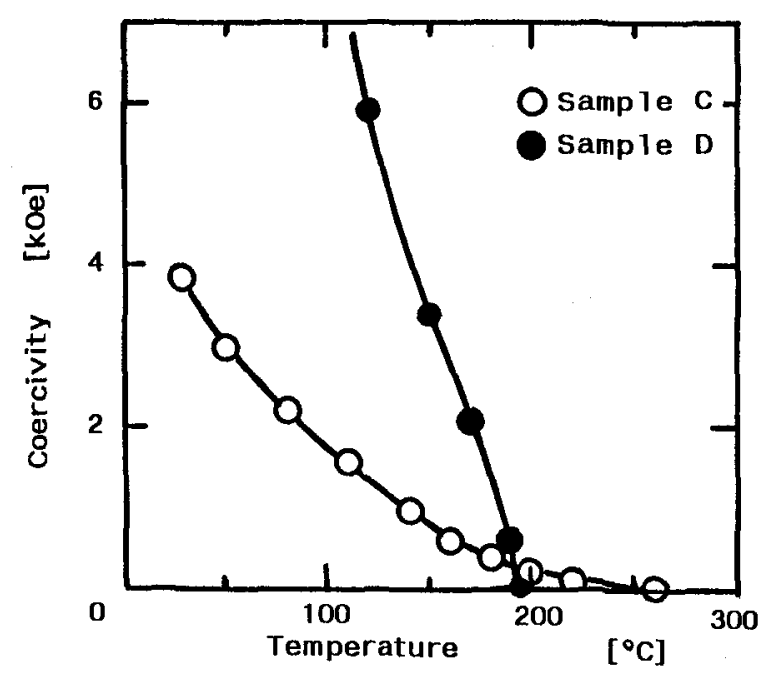

Fig. 7 Temperature dependence of Hc

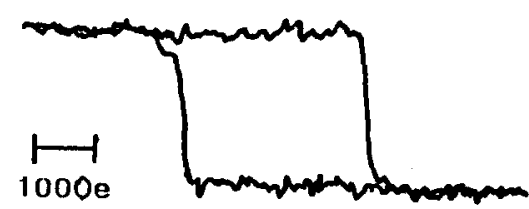

Fig.8 Kerr loop of sample $c$ at $200^{\circ}$ 